\title{
A convenient diagnostic tool for discriminating adult-onset glutamic acid decarboxylase antibody-positive autoimmune diabetes from type 2 diabetes : a retrospective study
}

\author{
Hon-Ke Sia ${ }^{1,2}{ }^{2}$, Shih-Te Tu ${ }^{1}$, Pei-Yung Liao ${ }^{1}$, Kuan-Han Lin ${ }^{2}$, Chew-Teng Kor ${ }^{3}$, Ling-Ling Yeh ${ }^{\text {Corresp. } 4}$ \\ ${ }^{1}$ Division of Endocrinology and Metabolism, Department of Internal Medicine, Changhua Christian Hospital, Changhua City, Taiwan \\ 2 Department of Healthcare Administration, Asia University, Taichung City, Taiwan \\ 3 Internal Medicine Research Center, Changhua Christian Hospital, Changhua City, Taiwan \\ 4 Social Enterprise and Innovation M.A. Program, Dharma Drum Institute of Liberal Arts, New Taipei City, Taiwan \\ Corresponding Author: Ling-Ling Yeh \\ Email address: YehLL@dila.edu.tw
}

Background. The glutamic acid decarboxylase antibody (GADA) test, commonly used to diagnose autoimmune diabetes, is not cost-effective in areas of low prevalence. The aim of this study was to develop a convenient tool to discriminate adult-onset GADA-positive autoimmune diabetes from type 2 diabetes (T2DM) in patients with newly diagnosed diabetes.

Methods. This retrospective cross-sectional study, conducted at Changhua Christian Hospital in Taiwan, collected electronic medical record data from January 2009 to January 2018. Patients were divided into a case group (GADA+, $n=152$ ) and a reference group (T2DM, $n=358$ ). Variables that differed significantly between the groups were subjected to receiver operator characteristic analysis to establish cutoff values. Discriminant function analysis was then employed to discriminate the two groups.

Results. At the onset of diabetes, the GADA+ group was younger, with lower body mass index (BMI), higher hemoglobin $\mathrm{Alc}(\mathrm{HbAlc})$, higher high-density lipoprotein cholesterol (HDL-C), and lower total cholesterol and triglycerides (TG). Five major factors were identified to form the linear discriminant functions: BMI, age at onset, TG, HDL-C, and HbAlc. BMI $<23 \mathrm{~kg} / \mathrm{m}^{2}$ was the most important factor, followed by TG $<98 \mathrm{mg} / \mathrm{dL}, \mathrm{HDL}-\mathrm{C} \geq 46 \mathrm{mg} / \mathrm{dL}$, age at onset $<30$ years, and $\mathrm{HbAlc} \geq 8.6 \%$. The overall accuracy of the linear discriminant functions was $87.1 \%$, with $84.2 \%$ sensitivity and $88.3 \%$ specificity.

Conclusions. Routine tests in diabetes care were used to establish a convenient, low-cost tool that may assist in the early identification of adult-onset GAD+ autoimmune diabetes in clinical practice. 
1 A convenient diagnostic tool for discriminating adult-onset glutamic acid decarboxylase

2 antibody-positive autoimmune diabetes from type 2 diabetes : a retrospective study

3

4 Hon-Ke Sia1,2, Shih-Te Tu1, Pei-Yung Liao1, Kuan-Han Lin², Chew-Teng Kor ${ }^{3}$, Ling-Ling $5 \quad \mathrm{Yeh}^{4}$

$7 \quad{ }^{1}$ Division of Endocrinology and Metabolism, Department of Internal Medicine, Changhua

8 Christian Hospital, 135 Nan-Hsiao Street, Changhua City 500, Taiwan

92 Department of Healthcare Administration, Asia University, 500 Lioufong Rd., Wufeng, $10 \quad$ Taichung City 41354, Taiwan

11 3nternal Medicine Research Center, Changhua Christian Hospital, 135 Nan-Hsiao Street, 12 Changhua City 500, Taiwan

$13{ }^{4}$ Social Enterprise and Innovation M.A. Program, Dharma Drum Institute of Liberal Arts, 14 No. 700, Fagu Rd., Jinshan Dist., New Taipei City, 20842, Taiwan

16 Corresponding Author:

$17 \quad$ Ling-Ling Yeh

18 No. 700, Fagu Rd., Jinshan Dist., New Taipei City, 20842, Taiwan

19 Email address: YehLL@dila.edu.tw

\section{Abstract}

22 Background. The glutamic acid decarboxylase antibody (GADA) test, commonly used to 23 diagnose autoimmune diabetes, is not cost-effective in areas of low prevalence. The aim of this 
24 study was to develop a convenient tool to discriminate adult-onset GADA-positive autoimmune 25 diabetes from type 2 diabetes (T2DM) in patients with newly diagnosed diabetes.

26 Methods. This retrospective cross-sectional study, conducted at Changhua Christian Hospital in 27 Taiwan, collected electronic medical record data from January 2009 to January 2018. Patients 28 were divided into a case group $(\mathrm{GADA}+, \mathrm{n}=152)$ and a reference group (T2DM, $\mathrm{n}=358)$.

29 Variables that differed significantly between the groups were subjected to receiver operator 30 characteristic analysis to establish cutoff values. Discriminant function analysis was then 31 employed to discriminate the two groups.

32 Results. At the onset of diabetes, the GADA+ group was younger, with lower body mass index 33 (BMI), higher hemoglobin A1c (HbA1c), higher high-density lipoprotein cholesterol (HDL-C), 34 and lower total cholesterol and triglycerides (TG). Five major factors were identified to form the 35 linear discriminant functions: BMI, age at onset, TG, HDL-C, and HbA1c. BMI $<23 \mathrm{~kg} / \mathrm{m}^{2}$ was 36 the most important factor, followed by $\mathrm{TG}<98 \mathrm{mg} / \mathrm{dL}, \mathrm{HDL}-\mathrm{C} \geq 46 \mathrm{mg} / \mathrm{dL}$, age at onset $<30$ 37 years, and $\mathrm{HbA} 1 \mathrm{c} \geq 8.6 \%$. The overall accuracy of the linear discriminant functions was $87.1 \%$, 38 with $84.2 \%$ sensitivity and $88.3 \%$ specificity.

39 Conclusions. Routine tests in diabetes care were used to establish a convenient, low-cost tool that may assist in the early identification of adult-onset GAD+ autoimmune diabetes in clinical 41 practice.

\section{Introduction}

44 In children and adolescents, type 1 diabetes (T1DM), also known as autoimmune diabetes, usually presents with diabetic ketoacidosis (DKA) as the initial presentation and is relatively 46 straightforward to diagnose. However, the clinical features of adult-onset autoimmune diabetes 
47 can be less typical. The period of insulin requirement after onset may allow differentiation

48 between patients with latent autoimmune diabetes in adults (LADA) and those with classic adultonset T1DM, who become insulin-dependent within 3 months of diagnosis [1]. LADA often involves slow, progressive islet destruction. In the absence of autoantibody testing, it is not uncommon for it to be diagnosed and treated as type 2 diabetes (T2DM) [1,2]. The lack of optimal treatment can result in deterioration of the autoimmune process, the acceleration of beta cell loss, faster progression to insulin dependence, and an increased risk of complications [2,3]. cytoplasmic autoantibodies (ICA), insulin autoantibodies (IAA), glutamic acid decarboxylase antibodies (GADA), tyrosine phosphatase IA-2 autoantibodies (IA-2A), and zinc transporter 8

57 autoantibodies (ZnT8A) are the major autoantibodies of clinical and research interest $[1,4]$. The reported incidence of T1DM-related autoantibodies in adult-onset diabetes is approximately 3\%$12 \%$, with variations between countries and ethnicities [1-3]. GADA are persistent in patients with long-standing diabetes and is not influenced by the age at disease onset. Therefore, the presence of GADA represents the most sensitive marker in adult-onset autoimmune diabetes [1]. However, the GADA test has not been widely used in primary care because of its cost and limited availability. In areas with a low prevalence of autoimmune diabetes, GADA measurement is not cost-effective as a routine test and can only be used in highly selective cases. It would therefore be desirable to establish a practical tool that could be used to determine whether to test GADA for suspected patients.

Fourlanos et al. proposed a LADA clinical risk score as a clinical screening tool to identify patients with LADA [5]. The parameters included age at onset $<50$ years, acute symptoms, body mass index $(\mathrm{BMI})<25 \mathrm{~kg} / \mathrm{m}^{2}$, and a personal or family history of autoimmune disease. 
70 According to a study by Shields, the two most important indicators for identifying T1DM and

71 T2DM are age at diagnosis $<35$ years and time to insulin $<6$ months [6]. These studies showed

72 that age at diagnosis and BMI were important discriminators. Conversely, Thunander et al.

73 demonstrated that C-peptide level at diagnosis was better than age or BMI in discriminating

74 between autoimmune and non-autoimmune diabetes [7]. However, C-peptide measurement is

75 also limited by its cost and availability.

76 The metabolic and clinical phenotypes of adult-onset autoimmune diabetes are

77 heterogeneous, ranging from patients who are lean and insulin sensitive to those who are obese

78 and insulin resistance [1]. There is evidence that the various disease processes observed in

79 subtypes of autoimmune diabetes can be detected by different autoantibodies. Diabetic patients

80 positive for the IA-2 $(256-760)$ autoantibody showed slow beta cell deterioration and clinical and

81 metabolic phenotype, similar to those with classic type 2 diabetes and obesity [1,3]. Conversely,

82 the A Diabetes Outcome Progression Trial (ADOPT) reported that GADA+ diabetic patients had

83 higher levels of serum high-density lipoprotein cholesterol (HDL-C) and lower levels of serum

84 triglycerides (TG) than those diagnosed T2DM, as well as a lower prevalence of metabolic

85 syndrome [8]. A Korean population study found that GADA levels were inversely associated

86 with age at onset, C-peptide levels, BMI, and total cholesterol and TG concentrations [9]. These

87 studies demonstrated that the lipid profile can help to distinguish autoimmune diabetes with

88 GADA + from T2DM.

89 Several related studies have used multiple islet autoantibodies to detect autoimmune

90 diabetes rather than focusing on GADA [1-3]. Subtype studies pointing to GADA+ diabetes are

91 therefore valuable in providing additive information for managing adult-onset autoimmune

92 diabetes. In addition, there is still some debate on the definition of LADA as well as on the 
93 classification of type 1 diabetes mellitus [2]. Using GADA+ diabetes in the present study would

94 help in defining the research subjects more distinctly and precisely.

95 The aim of the present study was to develop a convenient, cost-effective tool that can be used

96 to discriminate adult-onset GADA+ autoimmune diabetes from T2DM. Such a tool could help

97 physicians decide whether to perform a GADA test. To avoid additional costs, we based the tool

98 on parameters obtained from diabetes routine tests, such as lipid profile and hemoglobin A1c 99 (HbA1c).

100

101 Materials \& Methods

102 Subjects

103 This retrospective cross-sectional study was conducted at Changhua Christian Hospital

$104(\mathrm{CCH})$, a medical center in Taiwan. A total of 636 patients with diabetes who received the

105 GADA test between January 1, 2009 and December 31, 2018 were screened for eligibility based

106 on data from the hospital's electronic medical record system. Among these, 484 patients were

107 excluded for the following reasons: with GADA $<1.0 \mathrm{U} / \mathrm{mL}$, incomplete lipid and A1c data,

108 diabetes not diagnosed within the previous six months, age at onset $<20$ years, and excessive

109 alcohol consumption. Finally, 152 patients were enrolled into the case group (GADA+ group).

110 Excessive alcohol consumption was defined as consuming 7 drinks, amounting to approximately

11170 grams of alcohol, or more per week for both men and women.

112 The reference group (T2DM group) included 358 type 2 diabetic patients, newly diagnosed

113 between January 1, 2010 and December 31, 2013, with age at onset $\geq 20$ years. GADA was not a

114 routine test in the reference group. However, GADA testing was selectively performed for

115 patients suspected of having autoimmune diabetes in follow-up visits. Two diabetologists 
116 reviewed the medical records from January 1, 2010 to December 31, 2018, for each patient of the

117 reference group to confirm the diagnosis and to reduce the possibility of misclassification to the

118 maximum extent. Thus, 15 were excluded from the 406 patients identified for assessment (Figure

119 1).

120

121 Data collection

122 The data for both groups were obtained from the hospital's electronic medical record system.

123 Basic data (age at the onset of diabetes, and gender), personal habits (smoking and drinking),

124 height, and body weight were those collected at the initial visit for the diagnosis of diabetes. The

125 laboratory data used in the analysis, including $\mathrm{HbA1c}$, creatinine $(\mathrm{Cr})$, and glutamic pyruvic

126 transaminase (GPT) levels, were those measured closest to the first visit. The lipid profile

127 selected, including total cholesterol, HDL-C and TG levels, was that measured 2-12 weeks after

128 the first visit, documenting the use of any lipid-lowering agents (fibrates or statins) before the

129 lipid profile test.

130

131 Measurements

132 The diagnosis of diabetes mellitus was made according to the American Diabetes Association 133 criteria of 2019 [10]. Two diabetologists with more than five years' clinical experience reviewed 134 the medical records to validate the diagnosis. In cases of disagreement, the patient was excluded.

135 The total amount of alcohol consumption in grams per week was calculated from weekly alcohol 136 consumption (in millimeter) and the alcoholic concentration of each kind of beverage.

137 GADA was measured with an anti-GAD radioimmunoassay kit (RSR Ltd, Cardiff, UK).

138 According to the manufacturer, a serum concentration of GADA $\geq 1 \mathrm{u} / \mathrm{ml}$ was considered to be 
139 positive for GADA. The specificity of the GADA test for diagnosing adult autoimmune diabetes

140 can be as high as $98 \%$, with a sensitivity of about $70 \%-80 \%$ [11].

141 Serum biochemistry, including glucose, Cr, and GPT levels and the lipid profile, was

142 performed using a UniCel DxC 800 SynchronTM Clinical System (Beckman Coulter, Brea, CA,

143 USA). Serum HbA1c was measured by ion-exchange high-performance liquid chromatography

144 using a VARIANTTM II Turbo system.

145 BMI was calculated as the body weight in kilograms divided by height in meters squared. A

146 BMI of $23.0-24.9 \mathrm{~kg} / \mathrm{m}^{2}$ was classified as overweight and $\geq 25 \mathrm{~kg} / \mathrm{m}^{2}$ as indicating obesity,

147 according to the Regional Office for the Western Pacific standard [12].

148

149 Statistical analyzes

150 To establish a convenient diagnostic tool for discriminating the case group (GADA+) from

151 the reference group (T2DM), the major discriminating factors were first identified and then used

152 to form discriminant functions by discriminant function analysis.

153 Step 1: Differences between the two groups for selected variables were evaluated by

154 Student's t-test for continuous variables and chi-square test for categorical variables. The

155 continuous variables with statistical significance were tested for normal distribution using the

156 Kolmogorov-Smirnov test, and data with P-values of $<0.05$ were regarded as non-normally

157 distributed. The non-normally distributed variables were transformed to ordinary variables for

158 further analysis. A cutoff value for each of these variables was determined from the point on the

159 receiver operating characteristic (ROC) curve with the minimum distance to the upper left

160 corner, calculated as the square root of $\left[(1-\text { sensitivity })^{2}+(1-\text { specificity })^{2}\right]$.

161 Step 2: Discriminant function analysis using the enter method was then applied to establish

162 the major discriminating variables according to the discriminant loadings (with absolute values $>$ 
163 0.3) in the structure matrix. These major discriminating variables were then analyzed, again with

164 the enter method, to determine the standardized canonical discriminant function.

165 All the tests were two-tailed with a significance level of 0.05 . SPSS version 25 software

166 (IBM Corp., Armonk, NY, USA) was used for the analysis.

167

168 Ethics statement

169 The study was approved by the Institutional Review Board of Changhua Christian Hospital 170 (CCH IRB No: 190702). Informed consent was waived.

171

172 Results

173 This study included a total of 510 Chinese patients who were ethnically homogeneous. In the 174 GADA+ group, 84 patients $(55.3 \%)$ had higher GADA titers $(\geq 32 \mathrm{U} / \mathrm{mL})$ than the others. Table 1751 summarizes the clinical features of the patients at the time of diagnosis of diabetes. The 176 GADA+ group was significantly younger, with lower BMI, higher HbAlc and HDL-C levels, 177 and lower total cholesterol, TG and GPT levels at onset of DM. There were no differences

178 between the two groups in the gender ratio, Cr levels, prevalence of smoking, or the use of lipid179 lowering agents (fibrates or statins).

180 ROC curves were used to determine the cutoff values of these variables for discriminating 181 the patients in the GADA+ group, as follows: $\mathrm{HbAlc} \geq 8.6 \%$, HDL-C $\geq 46 \mathrm{mg} / \mathrm{dL}$, total 182 cholesterol $<183 \mathrm{mg} / \mathrm{dL}, \mathrm{TG}<98 \mathrm{mg} / \mathrm{dL}$, and GPT $\geq 22 \mathrm{U} / \mathrm{L}$ (Table 2). ROC curve figures are 183 shown in supplement file 1 (Figure S1).

184 The discriminant function analysis identified five major discriminating variables from the 185 structure matrix: BMI, age at onset, TG, HDL-C, and HbAlc. Conversely, use of statins, use of 
186 fibrates, GPT and total cholesterol were excluded because their discriminant loadings were $<0.3$.

187 The remaining five major variables (BMI, age at onset, TG, HDL-C, and HbA1c) were analyzed, 188 again by using the enter method, to form linear discriminant functions (Table 3 ). In order of the 189 absolute magnitude of the correlation within the function, BMI $<23 \mathrm{~kg} / \mathrm{m}^{2}$ was the most 190 important factor, followed by TG $<98 \mathrm{mg} / \mathrm{dL}$, HDL-C $\geq 46 \mathrm{mg} / \mathrm{dL}$, age at onset $<30$ years, and $191 \mathrm{HbA} 1 \mathrm{c} \geq 8.6 \%$. The overall accuracy of the linear discriminant functions in discriminating 192 GADA+ diabetes was $87.1 \%$ (Table 4 ). The sensitivity was $84.2 \%$ and the specificity reached $19388.3 \%$.

194

195 Discussion

196 This study demonstrated that measuring serum TG and HDL-C levels at the onset of disease 197 can be used to help discriminate GADA+ autoimmune diabetes from T2DM in adults. The 198 findings were consistent with those of previous studies that reported lower TG and higher HDL$199 \mathrm{C}$ levels in patients with autoimmune diabetes [8,9]. To the best of our knowledge, the present 200 study is the first to focus on TG and HDL-C combined with BMI, age at onset, and HbA1c to 201 develop a practical discrimination tool to discriminate adult-onset GADA+ autoimmune diabetes 202 from T2DM in ethnic Chinese patients. The LADA clinical risk score proposed by Fourlanos et 203 al. showed a sensitivity of $90 \%$ and specificity of $71 \%$ for detecting LADA diagnosed by GADA 204 [5]. A Swedish study used fasting C-peptide level $<0.7 \mathrm{nmol} / \mathrm{L}$ to identify autoimmune diabetes; 205 this showed a sensitivity of $89 \%$ and specificity of $66 \%$ [7]. In comparison with these previous 206 studies, the tool proposed in the present study focused on discriminating GADA+ rather than 207 LADA from T2DM. In other words, the target population of GADA+ patients in our study 
208 included patients with LADA and classic T1DM. The latter are usually leaner and younger at the 209 onset of diabetes.

210 Similar to those reported previously, age at diabetes onset and BMI were still major

211 discriminators of autoimmune diabetes. In contrast to the LADA clinical risk score of Fourlanos

212 et al., which uses BMI $<25 \mathrm{~kg} / \mathrm{m}^{2}$ as a cutoff value and does not include patients younger than 30

213 years [5], the present study classified both age and BMI into three levels to make the scoring

214 more precise and showed that BMI $<23 \mathrm{~kg} / \mathrm{m}^{2}$ and age at onset $<30$ would be better

215 discriminating features of GADA+ autoimmune diabetes in Chinese patients than BMI 23-25

$216 \mathrm{~kg} / \mathrm{m}^{2}$ and age $30-50$ years. The possible cause of the lower cutoff of BMI in our study may be

217 due to the different characteristics of study subjects. First, our study subjects included patients

218 with LADA and classic T1DM. Many studies have reported that LADA patients could often be 219 overweight or obese and that being overweight or obese is associated with an increased risk of 220 LADA $[13,14]$. Second, we enrolled subjects with age at onset $\geq 20$ years. This was different 221 from the studies that enrolled patients with LADA; the latter are often defined as age at onset $>$ 22230 years [1]. Third, the BMI values in our study were obtained from the initial weight data of 223 newly diagnosed diabetic patients who just received short-term treatment and did not regain their 224 weight fully. Finally, the risk of diabetes starts to increase at a BMI of approximately $23 \mathrm{~kg} / \mathrm{m}^{2}$ 225 in Chinese, which is lower than the WHO BMI cutoff value used to define an increase in the 226 incidence among Europeans [12,15]. Nevertheless, such a low cutoff may harbor the risk of not 227 being able to identify some patients with LADA, particularly in non-Asians and in areas of high 228 prevalence.

229 Autoimmune diabetes differs from T2DM in several respects. Its pathogenesis is primarily 230 based on insulin deficiency rather than insulin resistance. The present study showed that patients 
231 with GADA+ autoimmune diabetes exhibited higher serum HDL-C and lower TG levels, which

232 can be used to discriminate the condition from T2DM. Although hypertriglyceridemia may occur

233 in undertreated individuals with autoimmune diabetes due to hyperglycemia, intensive glucose

234 control normalizes the lipid abnormalities [16-17]. To reduce any interference from

235 hyperglycemia, the lipid data in the current study were collected about 2-12 weeks after

236 commencing treatment for acute hyperglycemia.

237 Patients with autoimmune diabetes have worse glycemic control than those with T2DM,

238 possibly due to the limited production of endogenous insulin [2]. The present study showed that 239 patients with GADA+ autoimmune diabetes had higher baseline HbA1c values at disease onset

240 than those with T2DM. Paradoxically, the GADA+ patients had healthier lipid levels despite the

241 higher baseline HbA1c values. Hence, baseline HbA1c was another significant discriminator in 242 our linear discriminant functions, in combination with age, BMI, TG, and HDL-C.

243 This study had several strengths. All the patients were newly diagnosed diabetes, thus their 244 blood lipid and HbAlc levels were less affected by lipid-lowering and anti-diabetic agents. We 245 also excluded patients who had a habit of drinking alcohol. Using the parameters identified in 246 this study within 3 months of the diagnosis of diabetes may help physicians to decide earlier 247 whether to seek a GADA test for a patient.

248 The study also had several limitations. First, GADA was not measured for the reference 249 group, so it cannot be ruled out that some of the patients in this group had LADA; however, the 250 possibility of misclassification was reduced by the confirmation from two diabetologists that all of the 251 patients in this group had T2DM. Second, this hospital-based study would inevitably exhibit 252 selection bias. The patients who did receive GADA testing were not representatives for the 253 overall diabetes population. Third, we did not exclude smokers from study participation. 
254 Although there was no significant difference in the distributions of smokers between the two 255 groups, smoking might reduce HDL levels and hence increase the probability of false negative 256 results [18]. Fourth, the incidence and clinical features of autoimmune diabetes may vary 257 between regions and ethnicities [1,3]. In this study, all the research subjects were Chinese; it 258 remains to be verified whether the findings can be applied to other ethnic groups. Fifth, although $25987.1 \%$ of original grouped cases could be correctly classified by the linear discriminant functions 260 developed in this study, these functions were obtained from limited samples; they should be 261 tested in an independent population to verify the validity of the proposed method. Finally, 262 although patients with LADA than those with T2DM showed a higher prevalence of family 263 history or personal history of related autoimmune diseases [5,19], we did not have complete data 264 to include them into our model. From another perspective, the tool requires less information than 265 that needed for calculating Fourlanos et al.'s LADA clinical risk score, as well as a lesser 266 requirement for judgment about acute symptoms at onset and a history of related autoimmune 267 diseases; therefore, our tool may be more efficient in clinical practice.

268 However, to date, due to the broad heterogeneity of LADA, islet-cell antibodies

269 measurement remains essential to decrease the number of misdiagnosis of diabetes. Moreover, to 270 detect GADA+ diabetic patients is not the final goal. The clinical problem concerned should be 271 whether insulin-dependency can be detected early by all means.

272

\section{Conclusions}

274 An early diagnosis of adult-onset autoimmune diabetes would avoid patients receiving 275 unnecessary oral medication, as well as helping to predict the disease course and reducing acute 276 events such as DKA. The present study established a convenient diagnostic tool based on data 
277 obtained from existing routine tests for diabetes care, including BMI, TG, HDL-C, age at onset, 278 and HbA1c, which may assist with the early identification of adult-onset GADA+ autoimmune 279 diabetes, avoiding extra medical costs and reducing the burden to patients.

280

281 References

282 [1] Buzzetti R, Zampetti S, Maddaloni E. Adult-onset autoimmune diabetes: current knowledge 283 and implications for management. Nat Rev Endocrinol 2017;13:674-86.

284 doi:10.1038/nrendo.2017.99

285 [2] Carlsson S. Etiology and pathogenesis of latent autoimmune diabetes in adults (LADA)

286 compared to type 2 diabetes. Front Physiol 2019 doi: 10.3389/fphys.2019.00320

287 [3] Pieralice S, Pozzilli P. Latent autoimmune diabetes in adults: A review on clinical 288 implications and management. Diabetes Metab J 2018;42:451-64.

289 [4] Winter WE, Schatz DA. Autoimmune markers in diabetes. Clinical Chemistry 2011;57:16829075.

291 [5] Fourlanos S, Perry C, Stein MS, Stankovich J, Harrison LC, Colman PG. A clinical screening 292 tool identifies autoimmune diabetes in adults. Diabetes Care 2006;29:970-5.

293 [6] Shields BM, Peters JL, Cooper C, Lowe J, Knight BA, Powell RJ, Jones A, Hyde CJ, 294 Hattersley AT. Can clinical features be used to differentiate type 1 from type 2 diabetes? A 295 systematic review of the literature. BMJ Open 2015;5:e009088. doi:10.1136/bmjopen-2015296009088

297 [7] Thunander M, Torn C, Petersson C, Ossiansson B, Fornander J, Landin-Olsson M. Levels of 298 C-peptide, body mass index and age, and their usefulness in classification of diabetes in relation 
299 to autoimmunity, in adults with newly diagnosed diabetes in Kronoberg, Sweden. Eur J

300 Endocrinol 2012;166:1021-9. doi: org/10.1530/EJE-11-0797

301 [8] ADOPT Study Group. Phenotypic characteristics of GAD $(+)$ recently diagnosed patients

302 with type 2 diabetes in North America and Europe. Diabetes 2004;53:3193-200. doi:

303 org/10.2337/diabetes.53.12.3193

304 [9] Roh MO, Jung CH, Kim BY, Mok JO, Kim CH. The prevalence and characteristics of latent 305 autoimmune diabetes in adults (LADA) and its relation with chronic complications in a clinical 306 department of a university hospital in Korea. Acta Diabetol 2013;50:129-34. doi:

$307 \quad 10.1007 / \mathrm{s} 00592-010-0228-\mathrm{y}$

308 [10] American Diabetes Association. Classification and diagnosis of diabetes: standards of 309 medical care in diabetes-2019. Diabetes Care 2019;42 Suppl 1:S13-28. doi: org/10.2337/dc19310 S002

311 [11] Wenzlau JM, Juhl K, Yu L, Moua O, Sarkar SA, Gottlieb P, Rewers M, Eisenbarth GS, 312 Jensen J, Davidson HW, Hutton JC. The cation efflux transporter ZnT8 (Slc30A8) is a major 313 autoantigen in human type 1 diabetes. Proc Natl Acad Sci USA 2007;104:17040-5.

314 [12] World Health Organization, Regional Office for the Western Pacific (WPRO),

315 International Association for the Study of Obesity, International Obesity Task Force. The Asia316 Pacific Perspective: redefining obesity and its treatment. Sydney, Health Communications 317 Australia Pty Ltd, 2000. https://apps.who.int/iris/handle/10665/206936

318 [13] Hjort R, Ahlqvist E, Carlsson PO, Grill V, Groop L, Martinell M, Rasouli B, Rosengren A, 319 Tuomi T, Åsvold BO, Carlsson1 S. Overweight, obesity and the risk of LADA: results from a 320 Swedish case-control study and the Norwegian HUNT Study. Diabetologia 2018;61(6):1333-43. 321 doi: $10.1007 / \mathrm{s} 00125-018-4596-0$ 
322 [14] Luk AOY, Lau ESH, Lim C, Kong APS, Chow E, Ma RCW, Chan JCN. Diabetes-related 323 complications and mortality in patients with young-onset latent autoimmune diabetes: a 14-year 324 analysis of the prospective Hong Kong Diabetes Register. Diabetes care 2019;42:1042-50.

325 [15] Ko G, Chan J, Cockram C, Woo J. Prediction of hypertension, diabetes, dyslipidaemia or 326 albuminuria using simple anthropometric indexes in Hong Kong Chinese. Int J Obes 327 1999;23:1136-42. doi: 10.1038/sj.ijo.0801043

328 [16] Subramanian S, Chait A. Hypertriglyceridemia secondary to obesity and diabetes. Biochim 329 Biophys Acta 2012;1821(5):819-25.

330 [17] Guy J, Ogden L, Wadwa RP, Hamman RF, Mayer-Davis EJ, Liese AD, D'Agostino R, 331 Marcovina S, Dabelea D. Lipid and lipoprotein profiles in youth with and without type 1 332 diabetes: the SEARCH for Diabetes in Youth case-control study. Diabetes Care 2009;32(3):41633320.

334 [18] Jain RB, Ducatman A. Associations between smoking and lipid/lipoprotein concentrations 335 among US adults aged $\geq 20$ years. J Circ Biomark 2018;7:1849454418779310. [19] Zampetti S, Capizzi M, Spoletini M, Campagna G, Leto G, Cipolloni L, Tiberti C, Bosi E, 337 Falorni A, Buzzetti R. GADA titer-related risk for organ-specific autoimmunity in LADA 338 subjects subdivided according to gender (NIRAD study 6). J Clin Endocrinol Metab 339 2012;97:3759-65. doi: 10.1210/jc.2012-2037 
Figure 1

Enrollment of participants in the study

Abbreviations: GADA: glutamic acid decarboxylase antibody; T2DM: type 2 diabetes mellitus; DKA, diabetic ketoacidosis. 


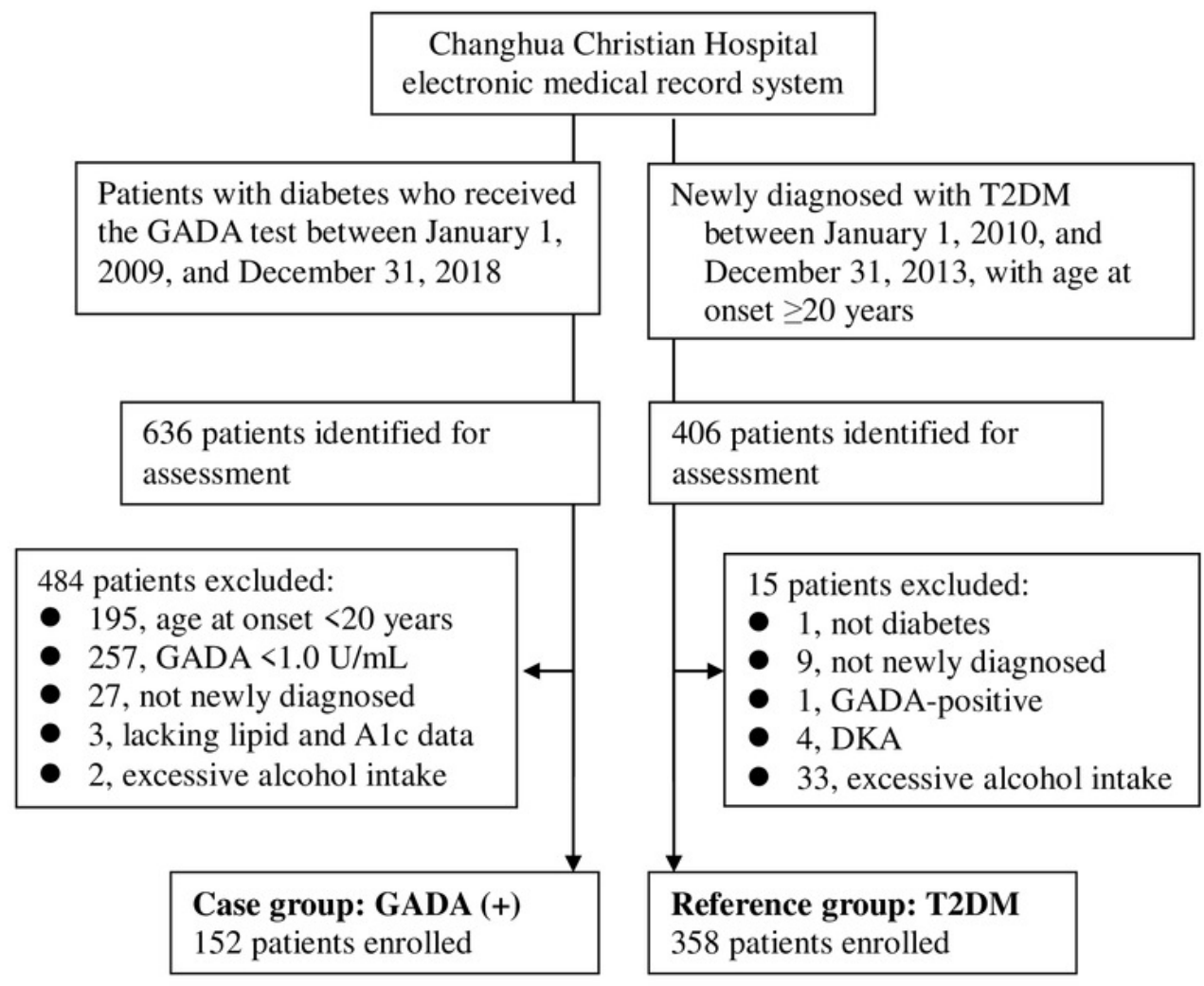




\section{Table $\mathbf{1}$ (on next page)}

Clinical features of the patients at the diagnosis of diabetes mellitus

${ }^{a}$ Chi-square test. ${ }^{b}$ Fisher's exact test. Other variables were examined by t-test.

Abbreviations: GADA+: Glutamic acid decarboxylase antibody-positive group; T2DM: type 2 diabetes mellitus group; BMI: body mass index; HDL-C: high-density lipoprotein cholesterol; TG: triglycerides; HbAlc: hemoglobin Alc; Cr: creatinine; GPT: glutamic pyruvic transaminase. 
1 Table 1. Clinical features of the patients at the diagnosis of diabetes mellitus

\begin{tabular}{|c|c|c|c|}
\hline & $\begin{array}{l}\text { GADA+ } \\
(\mathrm{n}=152)\end{array}$ & $\begin{array}{c}\text { T2DM } \\
(\mathrm{n}=358)\end{array}$ & \\
\hline & Mean $\pm \mathrm{SD}$ & Mean $\pm \mathrm{SD}$ & $p$ \\
\hline Gender: Men, n (\%) & $74(48.7)$ & $202(56.4)$ & $0.109^{\mathrm{a}}$ \\
\hline Age at onset (years) & $37.6 \pm 12.6$ & $50.4 \pm 11.7$ & $<0.001$ \\
\hline $20-29, \mathrm{n}(\%)$ & $48(31.6)$ & $18(5.0)$ & \\
\hline $30-49$, n $(\%)$ & $75(49.3)$ & $133(37.2)$ & \\
\hline$\geq 50, \mathrm{n}(\%)$ & $29(19.1)$ & $207(57.8)$ & \\
\hline BMI $\left(\mathrm{kg} / \mathrm{m}^{2}\right)$ & $21.7 \pm 3.8$ & $26.8 \pm 4.5$ & $<0.001$ \\
\hline$<23, \mathrm{n}(\%)$ & $114(75.0)$ & $62(17.3)$ & \\
\hline $23-24.9, \mathrm{n}(\%)$ & $16(10.5)$ & $70(19.6)$ & \\
\hline$\geq 25, \mathrm{n}(\%)$ & $22(14.5)$ & $226(63.1)$ & \\
\hline
\end{tabular}

Lipid profile

Total cholesterol (mg/dL)

$184.0 \pm 46.9$

$194.4 \pm 41.9$

0.014

$\mathrm{TG}(\mathrm{mg} / \mathrm{dL})$

$82.9 \pm 65.2$

$162.2 \pm 119.9$

$<0.001$

HDL-C (mg/dL)

$58.0 \pm 18.7$

$42.7 \pm 10.5$

$<0.001$

HbAlc (\%)

$10.5 \pm 3.2$

$8.2 \pm 2.3$

$<0.001$

GPT (U/L)

$25.1 \pm 18.7$

$35.2 \pm 29.2$

$<0.001$

Creatinine (mg/dL)

$0.8 \pm 0.2$

$0.8 \pm 0.2$

0.734

Smoking, n (\%)

33 (21.7)

64 (17.9)

$0.313^{\mathrm{a}}$

Use of statins, n (\%)

$5(3.3)$

$6(1.7)$

$0.317^{\mathrm{b}}$ 

Use of fibrates, n (\%)
$1(0.7)$
$5(1.4)$
$0.675^{\mathrm{b}}$

$2{ }^{a}$ Chi-square test. ${ }^{b}$ Fisher's exact test. Other variables were examined by t-test.

3 Abbreviations: GADA+: Glutamic acid decarboxylase antibody-positive group; T2DM: type 2 diabetes

4 mellitus group; BMI: body mass index; HDL-C: high-density lipoprotein cholesterol; TG: triglycerides;

5 HbA1c: hemoglobin A1c; Cr: creatinine; GPT: glutamic pyruvic transaminase. 


\section{Table 2 (on next page)}

Receiver operating characteristic analysis of variables for differentiating the GADA+ from the T2DM patients

${ }^{a}$ The minimum distance between a point on the receiver operating characteristic curve and the upper left corner, calculated as the square root of [(1-sensitivity $\left.)^{2}+(1 \text {-specificity })^{2}\right]$. The point with the minimum distance was used to define the cutoff value.

Abbreviations: GADA: Glutamic acid decarboxylase antibody; T2DM: type 2 diabetes mellitus; AUC: area under the receiver operating characteristic curve; $\mathrm{Cl}$ : confidence interval; HDL-C: high-density lipoprotein cholesterol; TG: triglycerides; HbAlc: hemoglobin Alc; GPT: glutamic pyruvic transaminase. 
1 Table 2. Receiver operating characteristic analysis of variables for differentiating the GADA+

2 from the T2DM patients

\begin{tabular}{lcccccc}
\hline \multicolumn{1}{c}{ Variables } & Cut-off & Sensitivity & Specificity & Minimum & AUC & $95 \%$ CI \\
& value & & & distance $^{\mathrm{a}}$ & \\
& & & & & \\
\hline HbA1c (\%) & 8.6 & $67.1 \%$ & $65.6 \%$ & 0.226 & 0.72 & $0.67-0.77$ \\
Total cholesterol (mg/dL) & 183 & $57.2 \%$ & $58.9 \%$ & 0.351 & 0.59 & $0.53-0.65$ \\
HDL-C (mg/dL) & 46 & $74.3 \%$ & $70.1 \%$ & 0.155 & 0.78 & $0.73-0.83$ \\
TG (mg/dL) & 98 & $77.6 \%$ & $70.7 \%$ & 0.136 & 0.80 & $0.76-0.85$ \\
GPT (U/L) & 22 & $55.3 \%$ & $65.6 \%$ & 0.318 & 0.66 & $0.60-0.71$
\end{tabular}

$3 \overline{{ }^{a}}$ The minimum distance between a point on the receiver operating characteristic curve and the upper left

4 corner, calculated as the square root of $\left[(1-\text { sensitivity })^{2}+(1-\text { specificity })^{2}\right]$. The point with the minimum

5 distance was used to define the cutoff value.

6 Abbreviations: GADA: Glutamic acid decarboxylase antibody; T2DM: type 2 diabetes mellitus; AUC:

7 area under the receiver operating characteristic curve; CI: confidence interval; HDL-C: high-density

8 lipoprotein cholesterol; TG: triglycerides; HbA1c: hemoglobin A1c; GPT: glutamic pyruvic transaminase. 


\section{Table 3 (on next page)}

Linear discriminant functions constructed from five major variables for discriminating GADA+ from T2DM patients

${ }^{a}$ Standardized canonical discriminant function: Wilks' lambda $=0.475, p<0.001$; eigenvalue 1.106; canonical correlation $=0.725$. Abbreviations: GADA+: Glutamic acid decarboxylase antibody-positive group; T2DM: type 2 diabetes mellitus group; BMI: body mass index; HDLC: high-density lipoprotein cholesterol; TG: triglycerides; HbAlc: hemoglobin Alc. 
1 Table 3. Linear discriminant functions constructed from five major variables for discriminating 2 GADA+ from T2DM patients

Variables

A

Age at onset $<30$ years

Age at onset: $30-50$ years

0.36

0.63

0.22

$-0.34$

0.32

0.36

0.43

$-0.11$

$-0.47$

2.525

2.442

0.30

2.442

4.658

2.808

(Constant)

$-7.917$

$-3.764$

3 a Standardized canonical discriminant function: Wilks' lambda $=0.475, \mathrm{p}<0.001$; eigenvalue 1.106;

4 canonical correlation $=0.725$.

5 Abbreviations: GADA+: Glutamic acid decarboxylase antibody-positive group; T2DM: type 2 diabetes

6 mellitus group; BMI: body mass index; HDL-C: high-density lipoprotein cholesterol; TG: triglycerides;

7 HbA1c: hemoglobin A1c. 


\section{Table 4 (on next page)}

Accuracy of the linear discriminant functions for detecting GADA+ patients

a. $87.1 \%$ of the original grouped cases were correctly classified Abbreviations: GADA+:

Glutamic acid decarboxylase antibody-positive group; T2DM: type 2 diabetes mellitus group. 
1 Table 4. Accuracy of the linear discriminant functions for detecting GADA+ patients

\begin{tabular}{lccc}
\hline & \multicolumn{2}{c}{ Predicted group membership } & \\
\cline { 2 - 3 } & GADA+ & Total \\
\hline GADA+ & $128(84.2 \%)$ & $24(15.8 \%)$ & $152(100 \%)$ \\
T2DM & $42(11.7 \%)$ & $316(88.3 \%)$ & $358(100 \%)$
\end{tabular}

2

3 a. $87.1 \%$ of the original grouped cases were correctly classified

4 Abbreviations: GADA+: Glutamic acid decarboxylase antibody-positive group; T2DM: type 2 diabetes

5 mellitus group. 